\title{
LIST OF PARTICIPANTS
}

\author{
Angelita L. Acebes \\ Entomology Department \\ Virginia Polytechnic Institute and State \\ University \\ Blacksburg, Virginia, 24061 USA \\ Karl Joy C. Acaba \\ Physics Department \\ Central Mindanao University \\ Musuan, Maramag, Bukidnon 8710 \\ Philippines
}

\section{Geneliza A. Galope}

Central Visayan Institute Foundation Jagna, Bohol 6308, Philippines

\author{
Alfonso Albano \\ Physics Department \\ Bryn Mawr College \\ Pennsylvania, USA \\ e-mail: alfonso.albano@gmail.com \\ Erwin P. Ambasa \\ Mathematics Department \\ Central Mindanao University \\ Musuan, Maramag, Bukidnon 8710 \\ Philippines
}

\section{Henry P. Aringa \\ Physics Department \\ Mindanao State University \\ Marawi City, Philippines \\ e-mail: henparinga@yahool.com}

\section{Dennis C. Arogancia}

Physics Department

MSU-Iligan Institute of Technology

Iligan City 9200, Philippines

\author{
John Paul J. Aseniero \\ Physics Department \\ Central Mindanao University \\ Musuan, Maramag, Bukidnon 8710 \\ Philippines
}

\author{
Rommel G. Bacabac \\ Medical Biophysics Group \\ Physics Department \\ University of San Carlos, Talamban, \\ Cebu City, Philippines \\ e-mail: rgbacabac@gmail.com

\section{Shiena Mae P. Balbon \\ Mathematics Department \\ Central Mindanao University \\ Musuan, Bukidnon 8710, Philippines \\ e-mail: xandra_max@yahoo.com}

\author{
Ryan B. Balili \\ Photonics Laboratory \\ Physics Department \\ MSU-Iligan Institute of Technology \\ Iligan City 9200, Philippines \\ e-mail: ryanbalili@yahoo.com
}

\section{Christopher C. Bernido}

Research Center for Theoretical Physics

Central Visayan Institute Foundation

Jagna, Bohol 6308, Philippines

e-mail: cbernido@mozcom.com

\section{Corazon C. Bernido}

Philippine Nuclear Research Institute

Commonwealth Ave., Diliman

Quezon City, Philippines

e-mail: cber823@yahoo.com 
Jinky B. Bornales

Physics Department

MSU-Iligan Institute of Technology

Iligan City 9200, Philippines

e-mail: jbornales@gmail.com

\section{Cecilia O. Bucayong}

Physics Department

Central Mindanao University

Musuan, Maramag, Bukidnon 8710,

Philippines

\section{Bienvenido M. Butanas Jr.}

Physics Department

Central Mindanao University

Musuan, Maramag, Bukidnon 8710,

Philippines

e-mail: bienbutz@yahoo.com

\section{Victoria Carpio-Bernido}

Research Center for Theoretical Physics

Central Visayan Institute Foundation

Jagna, Bohol 6308, Philippines

e-mail: cbernido@mozcom.com

\section{Karl Patrick Casas}

Cebu Normal University

Osmeña Boulevard, Cebu City 6000,

Philippines

\section{Leo Andrei A. Crisologo}

Philippine Science High School

Diliman, Quezon City, Philippines e-mail: leocrisologo@pshs.edu.ph

\section{Raymond S. Cruel}

Central Visayan Institute Foundation Jagna, Bohol 6308, Philippines

\section{Ryan John A. Cubero}

College of Marine Engineering

Department of Academics

Maritime Academy of Asia and the

Pacific, Kamaya Point,

Mariveles, Bataan 2105, Philippines

e-mail: cubero.ryan@yahoo.com

\section{Jonalizae P. Cuñado}

Central Visayan Institute Foundation Jagna, Bohol 6308, Philippines

\section{Vincent Daria}

Department of Quantum Science

Research School of Physics and

Engineering

Australian National University

Canberra, Australia

e-mail: vincent.daria@anu.edu.au

\section{Rolito G. Eballe}

Mathematics Department

Central Mindanao University

Musuan, Maramag, Bukidnon 8710,

Philippines

\section{Matthew George O. Escobido}

W. Sycip Graduate School of Business

Asian Institute of Management

123 Paseo de Roxas Ave.

Makati City 1260, Philippines

e-mail:mescobido@aim.edu

\section{Jose Manresa Enrico D. Espanol}

Philippine Science High School

Agham Road, Diliman, Quezon City, Philippines

\section{Hiroshi Ezawa}

Faculty of Science

Gakushuin University

Mejiro, Toshima-ku

Tokyo 171-8588, Japan

e-mail: hiroshi.ezawa@q.email.ne.jp

\section{Edward Aris D. Fajardo}

Physics Department

Mindanao State University

Marawi City, Philippines

\section{Josef Fröhlich}

Foresight and Policy Development

Austrian Institute of Technology

Tech Gate Vienna

Donau-City-Strasse 1

1220 Wien, Austria

e-mail: josef.froehlich@ait.ac.at

\section{Vicencia B. Galbizo}

Central Visayan Institute Foundation Jagna, Bohol 6308, Philippines 
Pablita C. Galos

Central Visayan Institute Foundation

Jagna, Bohol 6308, Philippines

\section{Beverly V. Gemao}

Physics Department

MSU-Iligan Institute of Technology

Iligan City 9200, Philippines

\section{Laksana Tri Handoko}

Research Center for Physics

Group for Theoretical and

Computational

Indonesian Institute of Sciences

Tangerang 15310, Indonesia

e-mail: handoko@handoko.net

\section{Jenneke Klein-Nulend}

Department of Oral Cell Biology

ACTA-University of Amsterdam and

VU University Amsterdam

Research Institute MOVE

Amsterdam, The Netherlands

e-mail: j.kleinnulend@acta.nl

\section{Tobias Kuna}

Department of Mathematics

University of Reading

PO Box 220, Reading, Berkshire

RG6 6AX, United Kingdom

e-mail: t.kuna@reading.ac.uk

\section{Reylan Loberternos}

Physics Department

University of San Carlos, Talamban,

Cebu City, Philippines

\section{Gibson Maglasang}

Cebu Normal University

Osmeña Boulevard, Cebu City 6000

Philippines

\section{Eduardo R. Mendoza}

Department of Computer Science

University of the Philippines, Diliman

Quezon City 1101, Philippines and

Max Planck Institute of Biochemistry

D-82152 Martinsried, Germany

e-mail: ermendoza@dcs.upd.edu.ph

\section{Toru Nakamura}

Faculty of Science

Gakushuin University

Mejiro, Toshima-ku

Tokyo 171-8588, Japan

e-mail: toru-nak@agate.plala.or.jp

\section{May Noren Nanud}

Physics Department

University of San Carlos

Talamban, Cebu City, Philippines

\section{Alviu Rey Nasir}

Physics Department

MSU-Iligan Institute of Technology

Iligan City 9200, Philippines

\section{Maria João Oliveira}

CMAF, University of Lisbon

P-1649-003 Lisbon, Portugal

oliveira@cii.fc.ul.pt

\section{Bhazel Anne R. Pelicano}

National Institute of Physics

University of the Philippines

Diliman, Quezon City 1101, Philippines bhazie@gmail.com

\section{Anthony M. Penaso \\ Central Mindanao University \\ Musuan, Maramag \\ Bukidnon 8710, Philippines \\ e-mail: anpen1972@yahoo.com \\ Lotis R. Racines \\ Physics Department \\ Central Mindanao University \\ Musuan, Maramag \\ Bukidnon 8710, Philippines}

Ruby Anna O. Rañoa

Central Visayan Institute Foundation Jagna, Bohol 6308, Philippines

\section{Normie Jean B. Sajor}

Physics Department

Central Mindanao University

Musuan, Maramag

Bukidnon 8710, Philippines 
James Salig, Jr.

Misamis University

H. T. Feliciano St., 7200 Ozamiz City, Philippines

e-mail: jamesboss2@gmail.com

\section{Ma. Esther O. Santos}

PLDT-Smart Foundation, Inc.

$7^{\text {th }}$ Floor Ramon Cojuangco Building

Makati Avenue corner dela Rosa St.,

Makati City, Philippines

\section{José Luís da Silva}

Centro de Ciencias Matemáticas

University of Madeira

P 9000-390 Funchal, Madeira, Portugal

e-mail: luis@uma.pt

\section{Ludwig Streit}

Research Center BiBoS

Universität Bielefeld, 33615 Bielefeld, Germany

and Centro de Ciencias Matemáticas

University of Madeira, P 9000-390

Funchal, Madeira, Portugal

e-mail: streit@pcserv.physik.uni-

bielefeld.de
Teresita D. Taganahan

Physics Department

Central Mindanao University

Musuan, Maramag

Bukidnon 8710, Philippines

\section{Janice Tutor}

Central Visayan Institute Foundation Jagna, Bohol 6308, Philippines

Frederik W. Wiegel

Institute of Theoretical Physics

University of Amsterdam

Valckenierstraat 65

1018 XE Amsterdam

The Netherlands

e-mail: f.w.wgl@hotmail.com 